\title{
CALENDER/MEETING NEWS
}

\section{MEDICAL IMAGING VII \\ SPIE 93}

Newport Beach Marriott Hotel and Tennis Club

Newport Beach, CA

February 14-19, 1993

For further information contact: Ms. Anne Noteboom, SPIE, telephone 206-676-3290.

\author{
THE THIRD PACS-RIS SCHOOL. \\ offered by \\ GEORGETOWN UNIVERSITY MEDICAL CENTER \\ and \\ BOWMAN GRAY SCHOOL OF MEDICINE \\ April 15-17, 1993 \\ Washington, DC
}

For further information contact: Susan Kirby, telephone 202-687-5990 or FAX 202-784-3479

\author{
IMAC '93 \\ ICC Berlin-International Congress Center \\ Berlin, Germany \\ June 23-24, 1993 \\ CAR '93 \\ Computer Assisted Radiology \\ Berlin, Germany \\ June 24-26, 1993
}

For further information contact: Prof. Heinz U. Lemke, Technische Universitat Berlin, Institut fur Technische Informatik, Sekr. CG FR 3-3, Franklinstrasse 28-29, D-1000 Berlin 10, GERMANY. Telephone $+49(\mathrm{O}) 30 / 314-73100$ or FAX $+49(\mathrm{O}) 30 / 314-21103$.

VISUAL COMMUNICATIONS AND IMAGE PROCESSING '93:

Eighth in a Series

Cambridge Hyatt Regency

Cambridge, MA

November 7-12, 1993

For exhibit information, please contact: Sue Davis, Director of Conferences and Exhibits at telephone 503/663-1284, FAX 503/663/9768. Or contact SPIE's Exhibit Department at telephone 206/6763290 , FAX 206/647-1445. 